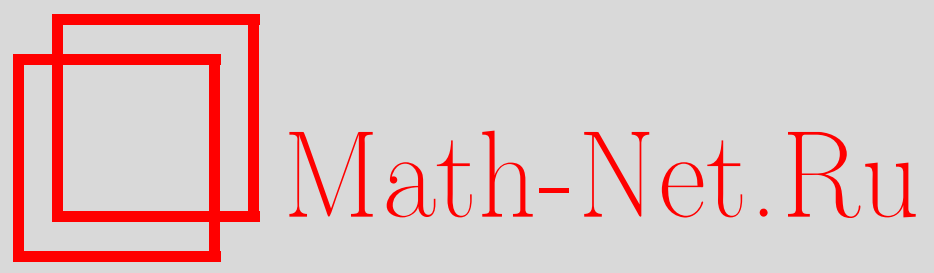

Обцероссийский математический портал

Х. Р. Мамедов, Единственность решения обратной задачи теории рассеяния для оператора Штурма-Лиувилля со спектральным параметром в граничном условии, Maтем. заметки, 2003, том 74, выпуск 1, 142-146

DOI: https://doi.org/10.4213/mzm587

Использование Общероссийского математического портала Math-Net.Ru подразумевает, что вы прочитали и согласны с пользовательским соглашением http://www. mathnet.ru/rus/agreement

Параметры загрузки:

IP : 52.90 .164 .192

26 апреля 2023 г., 18:19:40 


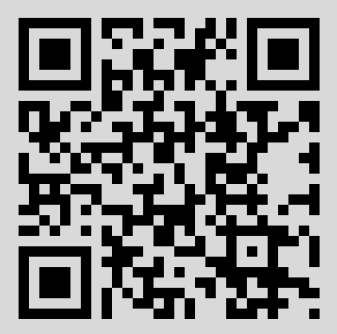




\section{ЕДИНСТВЕННОСТЬ РЕШЕНИЯ ОБРАТНОЙ ЗАДАЧИ ТЕОРИИ РАССЕЯНИЯ ДЛЯ ОПЕРАТОРА ШТУРМА-ЛИУВИЛЛЯ СО СПЕКТРАЛЬНЫМ ПАРАМЕТРОМ В ГРАНИЧНОМ УСЛОВИИ}

\section{Х. Р. Мамедов}

Рассмотрим на полуоси $0 \leqslant x<\infty$ краевую задачу, порожденную дифференциальным выражением

$$
-y^{\prime \prime}+q(x) y=\lambda^{2} y
$$

и краевым условием

$$
y^{\prime}(0)+\left(\alpha_{0}+i \alpha_{1} \lambda+\alpha_{2} \lambda^{2}\right) y(0)=0,
$$

где $\lambda$ - спектральный параметр, $q(x)$ - вещественная функция, удовлетворяющая условию

$$
\int_{0}^{+\infty}(1+x)|q(x)| d x<\infty
$$

а $\alpha_{0}, \alpha_{1}$ и $\alpha_{2}$ - вещественные числа, причем $\alpha_{1} \geqslant 0, \alpha_{2}>0$.

В данной работе доказьвается единственность решения обратной задачи теории рассеяния (ОЗТР) на полуоси для краевой задачи (1)-(3). Отметим, что ОЗТР для уравнения (1) с граничным условием, не содержащим спектральный параметр, полностью изучены в работах [1]- [3]. Обратные задачи на полуоси, связанные с уравнением (1), в различных постановках изучались во многих работах (см., например, [4]).

Известно (см. [2]), что при всех $\lambda$ из замкнутой верхней полуплоскости уравнение (1) имеет решение $e(x, \lambda)$, представимое в виде

$$
e(x, \lambda)=e^{i \lambda x}+\int_{x}^{\infty} K(x, t) e^{i \lambda t} d t
$$

При этом ядро $K(x, t)$ удовлетворяет соотношениям

$$
K(x, x)=\frac{1}{2} \int_{x}^{\infty} q(t) d t
$$

И

$$
|K(x, t)| \leqslant \frac{1}{2} \sigma\left(\frac{x+t}{2}\right) \exp \left\{\sigma_{1}(x)-\sigma_{1}\left(\frac{x+t}{2}\right)\right\}
$$

Работа поддержана The Scientific and Technical Research Council of Turkey (TUBITAK NATO PC-B). 
где положено $\sigma(x) \equiv \int_{x}^{\infty} q(t) d t, \sigma_{1}(x) \equiv \int_{x}^{\infty} \sigma(t) d t$. Свойства решений $e(x, \lambda)$ и $e(x,-\lambda)$ подробно изучены в [2]. Доказано, что $e(x, \lambda)$ является аналитической функцией от $\lambda$ в верхней полуплоскости и непрерывной функцией от $\lambda$ на вещественной оси. При этом во всей полуплоскости $\operatorname{Im} \lambda \geqslant 0$ справедливы оценки

$$
\begin{gathered}
|e(x, \lambda)| \leqslant \exp \left\{-\operatorname{Im} \lambda x+\sigma_{1}(x)\right\} \\
\left|e(x, \lambda)-e^{i \lambda x}\right| \leqslant\left\{\sigma_{1}(x)-\sigma_{1}\left(x+\frac{1}{|\lambda|}\right)\right\} \cdot \exp \left\{-\operatorname{Im} \lambda x+\sigma_{1}(x)\right\} \\
\left|e^{\prime}(x, \lambda)-i \lambda e^{i \lambda x}\right| \leqslant \sigma(x) \cdot \exp \left\{-\operatorname{Im} \lambda x+\sigma_{1}(x)\right\} .
\end{gathered}
$$

При вещественньх $\lambda \neq 0$ функции $e(x, \lambda)$ и $e(x,-\lambda)$ образуют фундаментальную систему решений уравнения (1), причем вронскиан этой системы равен

$$
W\{e(x, \lambda), e(x,-\lambda)\}=2 i \lambda .
$$

Обозначим через $\omega(x, \lambda)$ решения уравнения (1) при начальных данных

$$
\omega(0, \lambda)=1, \quad \omega^{\prime}(0, \lambda)=-\left(\alpha_{0}+i \alpha_{1} \lambda+\alpha_{2} \lambda^{2}\right) .
$$

Имеет место следующее утверждение.

Лемма 1. При вещественных значениях $\lambda \neq 0$ справедливо тождество

$$
\frac{2 i \lambda \omega(x, \lambda)}{e^{\prime}(0, \lambda)+\left(\alpha_{0}+i \alpha_{1} \lambda+\alpha_{2} \lambda^{2}\right) e(0, \lambda)}=\overline{e(x, \lambda)}-S(\lambda) e(x, \lambda)
$$

əəe

$$
S(\lambda)=\frac{\overline{e^{\prime}(0, \lambda)}+\overline{\left(\alpha_{0}-i \alpha_{1} \lambda+\alpha_{2} \lambda^{2}\right)} \overline{e(0, \lambda)}}{e^{\prime}(0, \lambda)+\left(\alpha_{0}+i \alpha_{1} \lambda+\alpha_{2} \lambda^{2}\right) e(0, \lambda)},
$$

причем

$$
\overline{S(\lambda)}=S(-\lambda)
$$

ЛЕмма 2. Функиия

$$
E(\lambda)=e^{\prime}(0, \lambda)+\left(\alpha_{0}+i \alpha_{1} \lambda+\alpha_{2} \lambda^{2}\right) e(0, \lambda)
$$

мохсет иметь в полуплоскости $\operatorname{Im} \lambda>0$ лишь конечное число нулей. При этом все эти нули простые и лежат на мнимой оси.

ДокаЗАТЕЛЬСтво. Так как при вещественных значениях $\lambda \neq 0$ справедливо неравенство $E(\lambda) \neq 0$, единственным вещественньм нулем функции $E(\lambda)$ может быть число $\lambda=0$. Из аналитичности функции $E(\lambda)$ в верхней полуплоскости следует, что ее нули образуют не более чем счетное множество.

Покажем, что это множество является ограниченньп. Допустим противное. Пусть существуют $\lambda_{k}$ такие, что $\left|\lambda_{k}\right| \rightarrow \infty$, при этом $\operatorname{Im} \lambda_{k}>0$ и $E\left(\lambda_{k}\right)=0$, т.е.

$$
e^{\prime}\left(0, \lambda_{k}\right)=-\left(\alpha_{0}+i \alpha_{1} \lambda_{k}+\alpha_{2} \lambda_{k}^{2}\right) e\left(0, \lambda_{k}\right)
$$

Тогда из оценки (7) при $\left|\lambda_{k}\right| \rightarrow \infty$ получаем, что $\lim _{k \rightarrow \infty} e\left(0, \lambda_{k}\right)=0$. С другой стороны, из (6) следует, что $\lim _{k \rightarrow \infty} e\left(0, \lambda_{k}\right)=1$. Полученное противоречие показьвает, что множество $\left\{\lambda_{k}\right\}$ ограничено.

Таким образом, нули функции $E(\lambda)$ образуют ограниченное и не более чем счетное множество, единственной предельной точкой которого может быть лишь нуль. 
Теперь покажем, что все нули функции $E(\lambda)$ лежат на мнимой оси. Пусть $\lambda_{1}$ и $\lambda_{2}$-какие-нибудь нули функции $E(\lambda)$. Умножая первое из соотношений

$$
\begin{aligned}
& -e^{\prime \prime}\left(x, \lambda_{1}\right)+q(x) e\left(x, \lambda_{1}\right)=\lambda_{1}^{2} e\left(x, \lambda_{1}\right), \\
& -\overline{e^{\prime \prime}\left(x, \lambda_{2}\right)}+q(x) \overline{e\left(x, \lambda_{2}\right)}=\overline{\lambda_{2}^{2}} \overline{e\left(x, \lambda_{2}\right)}
\end{aligned}
$$

на $\overline{e\left(x, \lambda_{2}\right)}$, а второе - на $e\left(x, \lambda_{1}\right)$, после чего вычитая второе из первого и интегрируя полученную разность от нуля до бесконечности, получим равенство

$$
\left(\lambda_{1}^{2}-\lambda_{2}^{2}\right) \int_{0}^{\infty} e\left(x, \lambda_{1}\right) \overline{e\left(x, \lambda_{2}\right)} d x-\left.W\left\{e\left(x, \lambda_{1}\right), \overline{e\left(x, \lambda_{2}\right)}\right\}\right|_{x=0}=0 .
$$

С другой стороны, согласно определению функции $E(\lambda)$ имеют место равенства

$$
E\left(\lambda_{j}\right)=e^{\prime}\left(0, \lambda_{j}\right)+\left(\alpha_{0}+i \alpha_{1} \lambda_{j}+\alpha_{2} \lambda_{j}^{2}\right) e\left(0, \lambda_{j}\right), \quad j=1,2 .
$$

Поэтому

$$
\begin{aligned}
& \left.W\left\{e\left(x, \lambda_{1}\right), \overline{e\left(x, \lambda_{2}\right)}\right\}\right|_{x=0}=e^{\prime}\left(0, \lambda_{1}\right) \overline{e\left(0, \lambda_{2}\right)}-e\left(0, \lambda_{1}\right) \overline{e^{\prime}\left(0, \lambda_{2}\right)} \\
& \quad=e\left(0, \lambda_{1}\right) \overline{e\left(0, \lambda_{2}\right)} \cdot\left(-\alpha_{0}-i \alpha_{1} \lambda_{1}-\alpha_{2} \lambda_{1}^{2}\right)-e\left(0, \lambda_{1}\right) \overline{e\left(0, \lambda_{2}\right)} \cdot\left(-\alpha_{0}+i \alpha_{1} \overline{\lambda_{2}}-\alpha_{2}{\overline{\lambda_{2}}}^{2}\right)
\end{aligned}
$$

откуда при $\lambda_{1}=\lambda_{2}$ следует, что

$$
\left.W\left\{e\left(x, \lambda_{1}\right), \overline{e\left(x, \lambda_{2}\right)}\right\}\right|_{x=0}=\left|e\left(0, \lambda_{1}\right)\right|^{2} \cdot\left[-\left(\overline{\lambda_{1}}+\lambda_{1}\right) i \alpha_{1}+\left({\overline{\lambda_{1}}}^{2}-\lambda_{1}^{2}\right) \alpha_{2}\right] .
$$

Следовательно, (10) принимает вид

$$
\left(\lambda_{1}+\overline{\lambda_{1}}\right)\left[\left(\lambda_{1}-\overline{\lambda_{1}}\right) \int_{0}^{\infty}\left|e\left(x, \lambda_{1}\right)\right|^{2} d x+\left(\lambda_{1}-\overline{\lambda_{1}}\right) \alpha_{2}|e(0, \lambda)|^{2}+i \alpha_{1}\left|e\left(0, \lambda_{1}\right)\right|^{2}\right]=0 .
$$

Так как $\lambda_{1}-\overline{\lambda_{1}} \neq 0$, из (11) следует, что $\lambda_{1}+\overline{\lambda_{1}}=0$, т.е. $\operatorname{Re} \lambda_{1}=0$. Значит, нули функции $E(\lambda)$ лежат только на мнимой оси.

Покажем теперь, что функция $E(\lambda)$ имеет конечное число нулей при $\operatorname{Im} \lambda \neq 0$. В общем случае конечность числа нулей функции $E(\lambda)$ вытекает из того, что можно дать оценку для расстояния между ее соседними нулями. Эта оценка может быть получена методом, приведенньм в [2]; при этом используется равенство (11).

Теперь покажем, что нули функции $E(\lambda)$ простые. Умножая первое из соотношений

$$
\begin{gathered}
-e^{\prime \prime}(x, \lambda)+q(x) e(x, \lambda)=\lambda^{2} e(x, \lambda), \\
-\dot{e}^{\prime \prime}(x, \lambda)+q(x) \dot{e}(x, \lambda)=2 \lambda e(x, \lambda)+\lambda^{2} \dot{e}(x, \lambda)
\end{gathered}
$$

на $\dot{e}(x, \lambda)$ (точка над $e(x, \lambda)$ означает производную по $\lambda$ ), а второе - на $e(x, \lambda)$, после чего вычитая первое соотношение из второго и интегрируя полученную разность от нуля до бесконечности, получим согласно определению функции $E(\lambda)$ и оценкам $(6),(8)$ равенство

$$
2 \lambda \int_{0}^{\infty}|e(x, \lambda)|^{2} d x=\left[\dot{E}(\lambda)-\left(i \alpha_{1}+2 \lambda \alpha_{2}\right) e(0, \lambda)\right] e(0, \lambda)-E(\lambda) \dot{e}(0, \lambda) .
$$

При помощи подстановки $\lambda=i \mu_{k}$ из последнего равенства получаем равенство

$$
2 \mu_{k} \int_{0}^{\infty}\left|e\left(x, i \mu_{k}\right)\right|^{2} d x+\left(\alpha_{1}+2 \alpha_{2} \mu_{k}\right)\left|e\left(0, i \mu_{k}\right)\right|^{2}=-i \dot{E}\left(i \mu_{k}\right) e\left(0, i \mu_{k}\right) .
$$

Следовательно, $\dot{E}\left(i \mu_{k}\right) \neq 0$, т.е. нули функции $E(\lambda)$ просты. Лемма доказана. 
Заметим, что согласно определению функции $E(\lambda)$ при $|\lambda| \rightarrow \infty$ справедлива асимптотика

$$
E(\lambda)=\alpha_{2} \lambda^{2}\left[1+O\left(\frac{1}{\lambda}\right)\right]
$$

Поэтому функция $S(\lambda)$, определенная формулой (9), которая называется функиией рассеяния уравнения (1) с начальньм условием (2), обладает при $|\lambda| \rightarrow \infty$ асимптотикой $S(\lambda)=1+O(1 / \lambda)$. Тем самым $1-S(\lambda) \in L_{2}(-\infty, \infty)$ и, значит, функция

$$
F_{s}(x)=\frac{1}{2 \pi} \int_{-\infty}^{\infty}(1-S(\lambda)) e^{i \lambda x} d \lambda
$$

тоже принадлежит пространству $L_{2}(-\infty, \infty)$.

Для вывода основного уравнения используем полученное в лемме 1 равенство (8):

$$
\frac{2 i \lambda \omega(x, \lambda)}{E(\lambda)}=\overline{e(x, \lambda)}-S(\lambda) e(x, \lambda)
$$

Подставляя сюда вместо $e(x, \lambda)$ его выражение $(4)$, получим

$$
\begin{aligned}
\frac{2 i \lambda \omega(x, \lambda)}{E(\lambda)}+2 i \sin \lambda x= & \int_{x}^{\infty} K(x, t) e^{-i \lambda t} d t+(1-S(\lambda)) e^{i \lambda x} \\
& +\int_{x}^{\infty} K(x, t)(1-S(\lambda)) e^{i \lambda t} d t-\int_{x}^{\infty} K(x, t) e^{i \lambda t} d t .
\end{aligned}
$$

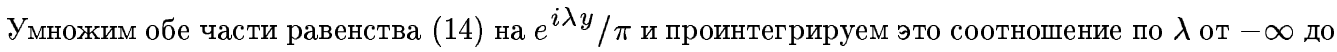
$+\infty$. В правой части при $y>x$ получим

$$
K(x, y)+F_{s}(x+y)+\int_{x}^{\infty} K(x, t) F_{s}(t+y) d t
$$

а в левой части, воспользовавшись леммой Жордана и теоремой о вычете, имеем

$$
-\sum_{k=1}^{n} \frac{2 i \lambda_{k} \omega\left(x, i \lambda_{k}\right) e^{-\lambda_{k} y}}{\dot{E}\left(i \lambda_{k}\right)}
$$

Учитывая формулу (12), можно преобразовать это выражение к такому виду:

$$
\begin{aligned}
& -\sum_{k=1}^{n} \frac{2 i \lambda_{k} \omega\left(x, i \lambda_{k}\right) e^{-\lambda_{k} y}}{\dot{E}\left(i \lambda_{k}\right)}=\sum_{k=1}^{n} \frac{2 \lambda_{k} e\left(x, i \lambda_{k}\right) e^{-\lambda_{k} y}}{i \dot{E}\left(i \lambda_{k}\right) e\left(0, i \lambda_{k}\right)} \\
& =-\sum_{k=1}^{n} \frac{2 \lambda_{k} e\left(x, i \lambda_{k}\right) e^{-\lambda_{k} y}}{2 \lambda_{k} \int_{0}^{\infty}\left|e\left(x, i \lambda_{k}\right)\right|^{2} d x+\left(\alpha_{1}+2 \alpha_{2} \lambda_{k}\right)\left|e\left(0, i \lambda_{k}\right)\right|^{2}}=-\sum_{k=1}^{n} m_{k}^{2} e\left(x, i \lambda_{k}\right) e^{-\lambda_{k} y} \\
& =\sum_{k=1}^{n} m_{k}^{2}\left\{e^{-\lambda_{k}(x+y)}+\int_{x}^{\infty} K(x, t) e^{-\lambda_{k}(t+y)} d t\right\}
\end{aligned}
$$

где

$$
m_{k}^{-2}=\int_{0}^{\infty}\left|e\left(x, i \lambda_{k}\right)\right|^{2} d x+\frac{\alpha_{1}+2 \alpha_{2} \lambda_{k}}{2 \lambda_{k}}\left|e\left(0, i \lambda_{k}\right)\right|^{2} .
$$

Итак, при $y>x$, учитывая (15) и (16), выводим из (14) равенство

$$
\begin{array}{r}
-\sum_{k=1}^{n} m_{k}^{2}\left\{e^{-\lambda_{k}(x+y)}+\int_{x}^{\infty} K(x, t) e^{-\lambda_{k}(t+y)} d t\right\} \\
=F_{s}(x+y)+\int_{x}^{\infty} K(x, t) F_{s}(t+y) d t+K(x, y)
\end{array}
$$


откуда окончательно получаем

$$
F(x+y)+K(x, y)+\int_{x}^{\infty} K(x, t) F(t+y) d t=0, \quad x<y<\infty,
$$

где

$$
F(x)=\sum_{k=1}^{n} m_{k}^{2} e^{-\lambda_{k} x}+F_{s}(x)=\sum_{k=1}^{n} m_{k}^{2} e^{-\lambda_{k} x}+\frac{1}{2 \pi} \int_{-\infty}^{\infty}(1-S(\lambda)) e^{i \lambda x} d \lambda .
$$

Уравнение (17) называют основным уравнением обратной задачи теории рассеяния.

Таким образом, доказана следующая теорема.

Теорема 1. При каждом $x \geqslant 0$ ядро $K(x, y)$ оператора преобразования (специального решения (4)) удовлетворяет основному уравнению (17).

Очевидно, чтобы составить основное уравнение, достаточно знать функцию $F(x)$. В свою очередь, чтобы найти функцию $F(x)$, достаточно знать только набор величин $\{S(\lambda)(-\infty<\lambda<\infty)$; $\left.\lambda_{k} ; m_{k}(k=1,2, \ldots, n)\right\}$. Набор величин $\left\{S(\lambda) ; \lambda_{k} ; m_{k}\right\}$ будем назьвать данными рассеяния краевой задачи (1), (2). Зная данные рассеяния, можно построить по формуле (18) функцию $F(x)$ и написать основное уравнение (17) относительно неизвестной функции $K(x, y)$. Решив это уравнение, найдем ядро $K(x, y)$ оператора преобразования, а значит, и потенциал

$$
q(x)=-\frac{1}{2} \frac{d}{d x} K(x, x) .
$$

ТЕОРема 2. При каждом фиксированном $x>0$ основное уравнение (17) имеет единственное решение $K(x, y) \in L_{1}[x, \infty)$.

ДокАЗАТЕльство. Переходная функция $F_{s}(x)$ обладает свойствами, аналогичными свойствам переходной функции для задачи без спектрального параметра в краевых условиях, а потому доказательство теоремы 2 следует из [2, теорема 3.3.1].

Автор выражает благодарность проф. И. М. Гусейнову за внимание к работе.

\section{СПИСОК ЦИТИРОВАННОЙ ЛИТЕРАТУРЫ}

1. Марченко В. А. // Докл. АН СССР. 1955. Т. 104. № 5. С. 695-698. 2. Марченко В. А. Операторы Штурма-Лиувилля и их приложения. Киев: Наукова думка, 1977. 3. Левитан Б. М. // Матем. заметки. 1975. Т. 17. № 4. С. 611-624. 4. Почейкина-Федотова Е. А // Изв. вузов. Матем. 1972. № 7. С. 75-84. 